\title{
Frequency of NFKBIA deletions is low in glioblastomas and skewed in glioblastoma neurospheres
}

\author{
Monica Patanè ${ }^{1}$, Paola Porrati ${ }^{1}$, Elisa Bottega ${ }^{1}$, Sara Morosini², Gabriele Cantini ${ }^{2}$, Vita Girgenti ${ }^{3}$, Ambra Rizzo $^{3}$, \\ Marica Eoli ${ }^{1}$, Bianca Pollo ${ }^{4}$, Francesca L Sciacca ${ }^{3}$, Serena Pellegatta ${ }^{1,2}$ and Gaetano Finocchiaro ${ }^{1,2^{*}}$
}

\begin{abstract}
The NF-kB family of transcription factors is up-regulated in inflammation and different cancers. Recent data described heterozygous deletions of the NF-kB Inhibitor alpha gene (NFKBIA) in about 20\% of glioblastomas (GBM): deletions were mutually exclusive with epidermal growth factor receptor (EGFR) amplification, a frequent event in GBM. We assessed the status of NFKBIA and EGFR in 69 primary GBMs and in corresponding neurospheres (NS). NFKBIA deletion was investigated by the copy number variation assay (CNV); EGFR amplification by CNV ratio with HGF; expression of EGFR and EGFRvIll by quantitative PCR or ReverseTranscriptase PCR. Heterozygous deletions of NFKBIA were present in 3 of 69 primary GBMs and, surprisingly, in 30 of 69 NS. EGFR amplification was detected in 36 GBMs: in corresponding NS, amplification was lost in 13 cases and reduced in 23 (10 vs 47 folds in NS vs primary tumors; $\mathrm{p}<0.001$ ). The CNV assay was validated investigating HPRT1 on chromosome $X$ in females and males. Results of array-CGH performed on 3 primary GBMs and 1 NS line were compatible with the CNV assay. NS cells with NFKBIA deletion had increased nuclear activity of p65 (RelA) and increased expression of the NF-kB target IL-6. In absence of EGF in the medium, EGFR amplification was more conserved and NFKBIA deletion less frequent point to a low frequency of NFKBIA deletions in GBM and suggest that EGF in the culture medium of NS may affect frequency not only of EGFR amplifications but also of NFKBIA deletions.
\end{abstract}

\section{Introduction}

Glioblastoma multiforme (GBM) is the highest grade glioma, according to World Health Organization classification, and has an annual incidence of 5 cases per 100,000 people [1,2]. In recent years, a huge effort was made to achieve a more thorough characterization of genetic and molecular signatures of GBM, facilitating the identification of new molecular targets and leading to a classification in four molecular subtypes: classical, mesenchymal, proneural and neural $[3,4]$. The classical subtype is mostly characterized by loss of chromosome 10 and amplification of the epidermal growth factor receptor gene $(E G F R)$.

\footnotetext{
* Correspondence: gaetano.finocchiaro@gmail.com

${ }^{1}$ Molecular Neuro-Oncology Unit, IRCCS Foundation "C.Besta" Neurological Institute, via Celoria 11, 20133 Milan, Italy

${ }^{2}$ Department of Experimental Oncology, IFOM-IEO Campus, via Adamello 16, 20139 Milan, Italy

Full list of author information is available at the end of the article
}

In the past years several studies have pointed to the importance of EGFR and NF-kB pathways in formation, growth and relapse of many tumor types, including GBM, and recent evidence suggests a cross-talk between these pathways [5-8].

NF- $\mathrm{kB}$ is a heterodimeric transcription factor formed by a family of Rel proteins, sharing a common Nterminal DNA binding region (RelA/p65, RelB and RelC), and by proteins that contain an ankyrin domain (p50, p100). NF-kB complexes are maintained inactive in the cytoplasm through interaction with their inhibitor $\mathrm{I} k B \alpha$, encoded by the NFKBIA gene located on chromosome 14q13.2. Most stimuli activate this pathway through phosphorylation of the IKK complex, which in turn phosphorylates $\mathrm{IkB} \alpha$, leading the inhibitor to degradation and allowing nuclear translocation of NF-kB. In the nucleus, NF-kB regulates the transcription of several genes involved in proliferation, survival, tissue invasion, inhibition of apoptosis and

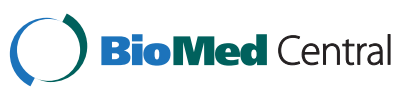


angiogenesis, including several chemokines and cytokines [9]. Two pathways of NF-kB activation have been described: canonical and non-canonical, involving different types of kinases (STAT3, PI3K/Akt, MAPK) and distinct heterodimers (p65/p50; p100/RelB) [10].

EGFR is mostly involved in proliferation and is expressed at high levels in many types of cancers, including glioblastoma [11]. EGFR is a tyrosine-kinase receptor, which signals through two main pathways: Ras/MAPK kinases and PI3K/Akt/mTOR kinases. Its gene, located on chromosome 7p11.2, is amplified in 40\% GBMs, forming typical double-minutes, auto-replicative chromosomes: this amplification is distinguished from polisomy of chromosome 7, a frequent event in GBM. $E G F R$ gene can be also mutated: in particular, the deletion of exons 2-7 generates a constitutively activated form, called EGFRvIII, which lacks of the extracellular domain and is not able to bind the EGF ligand and to internalize, leading to low-level continuous signalling. This mutant is present in $\sim 50 \%$ of GBM with EGFR amplification $[12,13]$ and reciprocal interactions between EGFR and EGFRvIII have been reported recently [14]. Another type of EGFR amplification was observed in $~ 30 \%$ of GBM with extra copies of the gene inserted in different loci of chromosome 7 ; in this form of amplification the number of gene copies is small and the percentage of amplified cells is less than $15 \%$ [15].

Several studies have pointed to a relationship between EGFR and NFKB pathways mostly through activation of PI3K/Akt/mTOR signalling $[7,16,17]$. Relevant interactions between EGFR, the most important oncogene in GBMs, and NF-kB have been first proposed in breast cancer [16]. In GBMs association between SHP-2 and Grb2-associated binder 1 (Gab1) was identified as a critical step in the pathway linking EGFR to NF-kB activation [18]. One report, in particular, proposed a correlation between NF-kB and EGFR status at the genetic level, describing the heterozygous deletion of NFKBIA in $20 \%$ of primary GBMs in mutual exclusion with EGFR amplification [19]. However, in a previous studiy based on single nucleotide polymorphism DNA microarray analysis of GBM we did not find evidence of chromosomal imbalance on chromosome 14q13.2, where NFKBIA is mapping [20].

We decided to investigate the NFKBIA status in relationship to EGFR in primary GBM and in GBM stemlike cells, a highly tumorigenic subpopulation exploiting stem cell programs for glioma perpetuation [21]. These cells are traditionally grown as neurospheres (NS) in the presence of EGF and bFGF, mimicking the growth of normal neural stem cells [22,23]. Surprisingly, we found that NFKBIA deletion is rare in primary tumors, but becomes frequent in GBM NS, an event that seems to be affected by the presence of EGF in the culture medium.

\section{Material and methods}

\section{Tumor specimens and cell cultures}

The research work described in this paper is in compliance with the Helsinki Declaration. Patients considered in this study had signed an informed consent, defined and approved by the Quality Office of the Istituto Neurologico Besta (CI 25), agreeing on the use of part of their tumor specimens for research purposes. Human glioblastoma specimens, diagnosed as grade IV gliomas according to WHO criteria, were snap frozen or paraffin-embedded after surgery. Neurospheres (here equivalent to glioblastoma stem-like cells) were derived by mechanical dissociation and digestion of tumor specimens with collagenase type I (Invitrogen, Life technologies, Foster City CA, USA). Single cell suspensions were plated at clonal density (50 cells/ $\mu \mathrm{l}$ ) in standard medium containing: DMEM/F-12 (GIBCO, Life Technologies, Foster City CA, USA), $2 \mathrm{mM}$ glutamine (Sigma Aldrich, St.Louis MO, USA), penicillinstreptomycin (1:100, EuroClone, Italy), B-27 supplement (1:50, GIBCO, Life technologies, Foster City CA, USA), human recombinant fibroblast growth factor 2 (bFGF $20 \mathrm{ng} / \mathrm{ml}$; Tebu Bio, France) and epidermal growth factor (EGF, 20 ng/ml; Tebu Bio, France).

\section{Nucleic acid extraction}

Total DNA was extracted using QIAamp DSP DNA Blood Mini Kit (Qiagen, Hilden, Germany) for peripheral blood and PureLink ${ }^{\text {mi }}$ Pro 96 Genomic DNA Purification Kit (Invitrogen, Life technologies, Foster City CA, USA) for paraffin-embedded tumors according to protocols of the manufacturers. Total DNA and RNA from neurospheres were extracted using All Prep DNA/RNA mini kit (Qiagen, Hilden, Germany). RNA from snap frozen specimens was extracted by TRIZOL ${ }^{\bullet}$ (Invitrogen, Life technologies, Foster City CA, USA) using the manufacturer's protocol. DNA and RNA concentration and purity were measured using Nanodrop ${ }^{\circ}$ (Thermo Scientific, Pierce Biotechnology, Rockford IL, USA).

\section{Copy number variation assay}

Copy number variation assay was performed on $\mathrm{ViiA}^{\mathrm{mm}} 7$ Real-Time PCR System (Applied Biosystems, Life technologies, Foster City CA, USA) using Taqman ${ }^{\circ}$ copy number assay chemistry (Applied Biosystems, Life technologies, Foster City CA, USA). Duplex real-time PCR was performed using $5 \mathrm{ng} / \mu \mathrm{l}$ of DNA. FAM-labeled assays were used for EGFR (Hs4942325_cn), HGF (Hs04982672_cn), NFKBIA (Hs01379535_cn), HPRT1 (Hs05654996_cn) (Applied Biosystems, Life technologies, Foster City CA, USA) and VIC-labeled assay was used for reference gene (TERT 20X, Applied Biosystems, Life technologies, Foster City CA, USA). DNA extracted from peripheral blood was used as control. To discriminate between EGFR amplification and polysomy of 
chromosome 7, EGFR copy number was normalized against HGF copy number also mapping on chr7 and usually not amplified: threshold for amplification was $E G F R / H G F$ copy number ratio $\geq 4$.

\section{RT-PCR and real time PCR}

500 ng RNA were reverse-transcribed using the High Capacity cDNA Reverse Transcription kit on 2720 Thermal Cycler (Applied Biosystems, Life technologies, Foster City CA, USA).

Real Time PCR was performed using Taqman ${ }^{\bullet}$ reagents on ViiA ${ }^{\text {тм }} 7$ Real-Time PCR System (Applied Biosystems, Life technologies, Foster City CA, USA): Taqman Universal Master Mix, EGFR assay (Hs_01076078_m1) and GAPDH assay as calibrator (Hs_99999905_m1) (Applied Biosystems, Life technologies, Foster City CA, USA). FirstChoice ${ }^{\bullet}$ Human Brain Total RNA was used as control (Ambion, Life technologies, Foster City CA, USA).

Amplification of full length EGFR and EGFRvIII was performed using Taq Gold ${ }^{\odot}$ DNA Polymerase on 2720 Thermal Cycler (Invitrogen, Life technologies, Foster City CA, USA). PCR conditions were as follows: $95^{\circ} \mathrm{C}$ for $10 \mathrm{~min} ; 42 \times\left[95^{\circ} \mathrm{C}\right.$ for $30 \mathrm{sec}, 56.5^{\circ} \mathrm{C}$ for $30 \mathrm{sec}$, and $72^{\circ} \mathrm{C}$ for $1 \mathrm{~min} 20 \mathrm{sec} \mathrm{sec}$ ]; and $72^{\circ} \mathrm{C}$ for $10 \mathrm{~min}$. PCR products were analyzed into a $2.0 \%$ agarose gel. The following primers were used: EGFRvIII forward 5'-CTTCGGGGAG CAGCGATGCGA-3' and reverse 5' -ACCAATACCTATT CCGTTACA-3'; wtEGFR forward 5'-CCAGTATTGATC GGGAGAGC-3' and reverse 5' - CCAAGGACCACCTCA CAGTT-3' (PRIMM, Italy).

\section{Evaluation of NF-kB activity and IL- 6 levels}

Cytoplasmatic and nuclear proteins were extracted using the Nuclear extract kit (Active Motif, Carlsbad CA, USA) according to manufacturer's protocol. Protein concentration was measured by MicroBCA Protein Assay kit at $\mathrm{OD}=540 \mathrm{~nm}$ (Thermo Scientific, Pierce Biotechnology, Rockford IL, USA) and analyzed using a microplate absorbance reader Multiskan FC (Thermo Scientific, Pierce Biotechnology, Rockford IL, USA).

NF-kB activity was measured in protein extracts using the TransAM ${ }^{\mathrm{TM}}$ NF-kB p65 protein assay (Active Motif, Carlsbad CA, USA), an ELISA-based method in which an oligonucleotide representing the NF-kB consensus binding site is immobilized on 96-well plates; p65 subunit is revealed by HRP-conjugated antibodies and analyzed at $\mathrm{OD}=450 \mathrm{~nm}$.

Interleukin-6 levels were measured from supernatants of neurospheres using Human IL-6 ELISA kit 96-well plate, based on standard sandwich enzyme-linked immuneadsorbent technology and revealed by Avidin-BiotinPeroxidase Complex System (Wuhan Boster Biological Technology, Wuhan China), according to manufacturer's protocol and analyzed at OD $=450 \mathrm{~nm}$.

\section{Array-CGH}

Array-CGH was performed using the CytoChip oligoarray (ISCA) 180K, containing 181,873 oligonucleotide probes (BlueGnome Ldt, Cambridge, UK) with a mean resolution of $16.30 \mathrm{~Kb}$ ( $25 \mathrm{~Kb}$ resolution on the backbone, $3.4 \mathrm{~Kb}$ resolution on genes). Data analysis was performed using InnoScan 710 Microarray Scanner (Innopsys Inc. Chicago IL, USA) and Bluefuse software (BlueGnome Ldt, Cambridge, UK). Oligo positions are referred to hg19.

One $\mu \mathrm{g}$ of sample and reference genomic DNAs were digested and then labelled with Cy3 (samples) and Cy5 (references) fluorophores, using random primers. The labelled DNAs were cleaned up using Amicon Ultracel-30 membrane filters (AU-30) (Millipore, Billerica MA, USA) followed by vacuum concentration if required. Labelled DNAs of samples were combined with sexmatched labeled references and then hybridized with oligonucleotide probes of array platform. Hybridization was performed using MaiTaiTM Hybridization System (SciGene, Sunnivale CA, USA). After 24 hours CytoChip oligo-array was washed and scanned. Amplifications or deletions are revealed by green $(\mathrm{Cy} 3)$ or red (Cy5) signals, due to unbalanced ratio between the two fluorophores.

Probes located at +0.3 and -0.3 in the hg19 are in double copy (normal genotype); probes at +0.40 and +0.60 are in triple copy; more than +0.60 it is referred to gene amplification. Probes at -0.6 and 1 are in single copy, whereas probes at -2 are nullisomic.

\section{Immunohistochemistry}

Surgical specimens are fixed in Carnoy, paraffin-embedded and sectioned at $2 \mu \mathrm{m}$. Sections are mounted on slides, paraffin removed by xylene and blocked in $10 \% \mathrm{H}_{2} \mathrm{O}_{2}$ (Sigma-Aldrich, St. Louise, Missouri, USA). The sections are first incubated with normal goat serum (Dako, Glostrup, Denmark) and then in a humidified chamber with mouse monoclonal anti-EGFR (Thermo Scientific, INC, USA). Sections are then incubated with anti-mouse Envision ${ }^{\bullet}$ peroxidase conjugated (Dako, Glostrup, Denmark). Finally, sections reacted with diaminobenzidine (Liquid DAB Substrate Chromogen System, DakoCytomation Carpinteria, CA, USA), counterstained with hematoxylin and mounted.

For hematoxylin-eosin staining, slides deparaffined in xylene are stained in Carazzi hematoxylin solution, rinsed in running tap water and counterstain in eosin solution.

\section{Data analysis}

The $\Delta \Delta \mathrm{Ct}$ method was used to determine gene expression and copy number variation; copy number variation $(\mathrm{CNV})$ was determined as $2\left(2^{-\Delta \Delta \mathrm{Ct}}\right)$, where 2 is the usual copy number of a gene [24]. Numerical results 
were expressed as means \pm SEM and statistical significance $(\mathrm{P}<0.05)$ was evaluated using Student $t$ test.

\section{Results}

\section{Clinical and histological features}

Tumor samples were obtained from 69 patients (48 males) operated at the IRCCS Foundation "C.Besta" Neurological Institute from 2007 to 2012. Mean age was 57 (range 18-78 years). 40/69 patients died after first surgery, 14/69 were re-operated after few months: the median survival time ranged from 9 to 12 months after surgery. Histologically, all tumors showed features of glioblastoma such as pleomorphic cells, microvascular proliferation, necrotic areas and pseudo-palisades.

\section{EGFR status in primary GBM and corresponding neurospheres}

We performed a copy number variation (CNV) assay to assess the EGFR amplification status in 69 primary GBM (here defined as brain tumors, BT) and in corresponding neurospheres (here defined as NS), cultured in the presence of EGF and bFGF. To differentiate EGFR amplification from polisomy of chromosome 7 we considered the ratio between $\mathrm{CNV}$ of EGFR and $H G F$, both located on chromosome 7, normalised to the reference gene TERT.

Table 1A summarizes results in brain tumors (BT) with or without EGFR amplification and in corresponding NS: EGFR was amplified in 36 tumors (52\%), but only in 23 corresponding NS (33\%).

Figure 1A shows the EGFR/HGF ratio: in BT the median CNV ratio was 47; this value significantly decreased in NS, with median CNV ratio of $10(\mathrm{p}<0.001)$.

Thus, 13/36 BT lost EGFR amplification in corresponding NS and EGFR CNV significantly decreased in the remaining $23 \mathrm{NS}$ when compared to BT values.

\section{NFKBIA status in primary GBM and corresponding neurospheres}

We then performed the $\mathrm{CNV}$ assay to assess the NFKBIA status on same BT and NS tested for EGFR amplification. Only 3 BT showed heterozygous deletion

\begin{tabular}{|c|c|c|c|}
\hline $1 \mathrm{~A}$ & EGFR wt & EGFR amp & $\%$ amp \\
\hline BT & 33 & 36 & $52 \%$ \\
\hline NS & 46 & 23 & $33 \%$ \\
\hline $1 \mathrm{~B}$ & NFKBIA wt & $N F K B I A+/-$ & $\%$ del \\
\hline BT & 66 & 3 & $4 \%$ \\
\hline NS & 39 & 30 & $43 \%$ \\
\hline $1 C$ & EGFR wt & EGFR amp & loss of EGFR amplification \\
\hline$N F K B I A+/-\mathrm{NS}$ & 11 & 12 & 7 \\
\hline
\end{tabular}

of NFKBIA and this alteration was mutually exclusive with $E G F R$ amplification. Surprisingly, however, heterozygous deletion of $N F K B I A$ was detected in a much larger number of cases, 30 of 69 NS lines (Table 1B).

The deletion was mutually exclusive with EGFR amplification in 18/30 NS; notably, 7/30 of these NS had lost EGFR amplification in vitro; in the remaining $12 \mathrm{NS}$ the heterozygous deletion was present concurrently with EGFR amplification (Table 1C).

Figure $1 \mathrm{~B}$ displays the median copy number of NFKBIA: combining BT and NS that we defined as hemizygously deleted for $N F K B I A$ the median $C N V$ was 1.2 , while in remaining $\mathrm{BT}$ and $\mathrm{NS}$ the median $\mathrm{CNV}$ was 2.6.

To confirm that the CNV assay is able to distinguish one from two copies of a gene, we performed an assay to evaluate HPRT1 CNV on 6 controls, 3 females and 3 males: HPRT1 maps on chromosome $\mathrm{X}$ and therefore one copy only is present in males. Accordingly, females had 2 copies of the gene and males one copy only (Additional file 1: Figure S1), confirming that the CNV assay used to study NFKBIA detects hemizygosity.

Validation of CNV data also derived from array-CGH experiments on four GBM: BT418, 419, 314 and NS297. EGFR amplification on 7p11.2 (probe position according to hg19 at +0.60) was confirmed in two primary BT (BT418 e BT419) in which we found high $E G F R / H G F$ ratios (74 and 323, respectively; BT418 shown in Figure 2C). BT314 showed trisomy of chromosome 7 and absence of EGFR amplification (probe position at +0.40$)$ in agreement with results of the CNV assay (Figure 2A). BT314 showed NFKBIA deletion in the primary tumor: this was confirmed by array$\mathrm{CGH}$, showing complete loss of the long arm of chromosome 14 (Figure 2B). In NS297 EGFR amplification was pinpointed by the $\mathrm{CNV}$ assay but not by array-CGH, suggesting the possibility of a focal intragenic deletion not detectable by the CGH probes used in this array that only flank NFKBIA.

Taken together the data confirm that the CNV assay is able to detect gene amplifications and heterozygous deletions of EGFR and NFKBIA, respectively.

\section{Functional validation of NFKBIA heterozygous deletion}

To validate further $\mathrm{CNV}$ data, we investigated the functional consequences of NFKBIA deletion. We speculated that the deletion could decrease intracellular levels of the NF-kB inhibitor (IkB), causing increased translocation of NF-kB into the nucleus and, consequently, increased transcription of target genes.

As a readout of NF-kB activity we investigated p65 subunit activation. We compared p65 activity in cytoplasmic and nuclear extracts of $8 \mathrm{NS}$ with NFKBIA deletion and 6 without deletion. As shown in Figure 3, we found that p65 activity was significantly higher in 


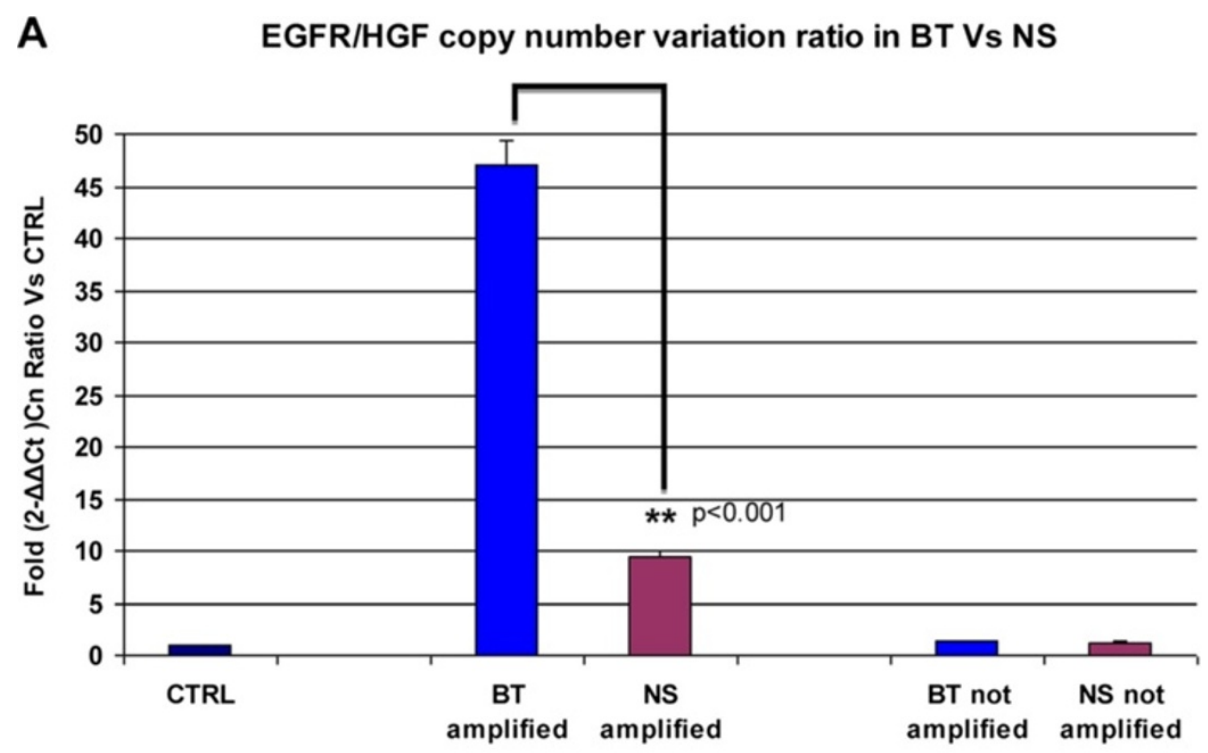

\section{B NFKBIA copy number variation in BT Vs NS}

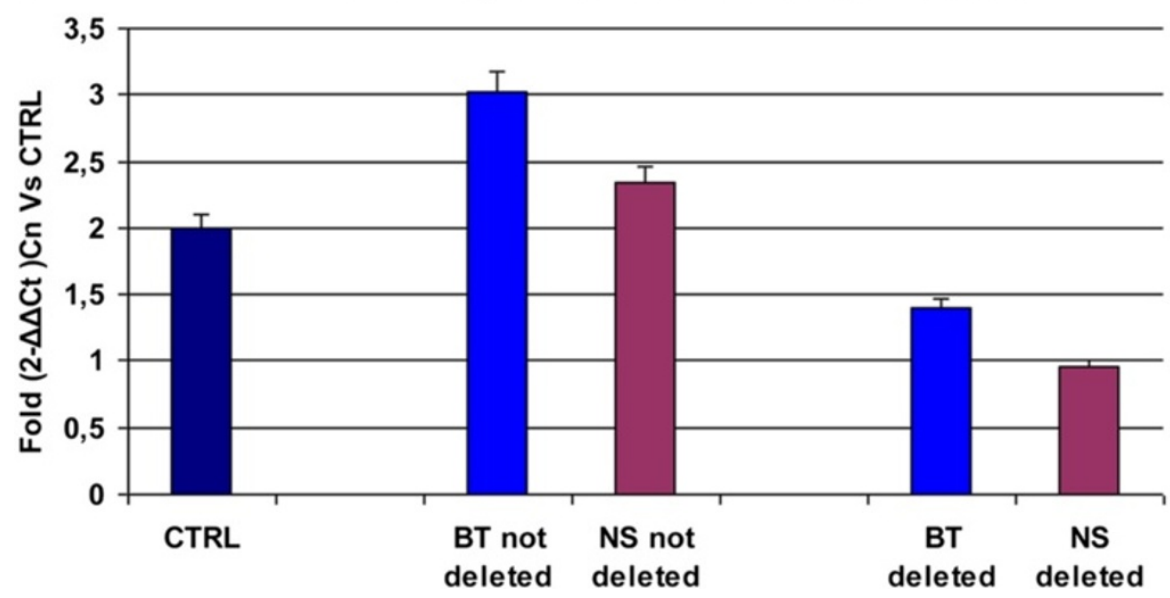

Figure 1 Copy number variation values (CNVs) in primary GBM (brain tumors, BT) and corresponding neurospheres (NS). A) EGFR amplification in BT and corresponding NS is expressed as EGFR/HGF ratio in comparison to control DNA and shows significant loss of amplification in vitro $\left({ }^{* *} \mathrm{p}<0.001\right)$. B) Heterozygous deletion in BT and NS shows a median copy number value of 1.2 compared to healty DNA and not deleted lines.

nuclear extracts of NS with NFKBIA deletion than in others $(\mathrm{p}<0.005)$. This observation confirms that NFKBIA deletion in GBM NS may have relevant functional consequences.

As a further readout of NF-kB activity we also measured IL-6 levels in the culture medium of NS 462 and NS 470. This pro-inflammatory cytokine is a known target of NF-kB transactivation. Notably, IL-6 is also produced in EGFRvIII cells and released in the microenvironment to activate adjacent wtEGFR cells through GP130 activation [25]. IL-6 levels showed correlation with the presence of the EGFRvIII variant and NFKBIA deletion: they were present in NS 462 cells, cultured with or without EGF and in NS 470 cells cultured in complete medium, but were very low in the absence of
EGF, when EGFRvIII is almost absent and NFKBIA is not deleted (Figure 4).

\section{Characterization of NS cultured with or without EGF}

Previous data showed that EGF in the culture medium of NS may affect EGFR amplification [26]. To evaluate the contribution of EGF in the culture medium to EGFR amplification and /or NFKBIA expression, we established NS cultures from primary GBMs in the presence of either complete or modified medium (i.e. containing both EGF and bFGF or bFGF only, respectively).

We first investigated four NS lines deriving from BT with $E G F R$ amplification. Figure 5 shows the comparison of EGFR/HGF copy number ratio (Figure 5A) and NFKBIA copy number (Figure $5 \mathrm{~B}$ ) in BT, NS in complete 


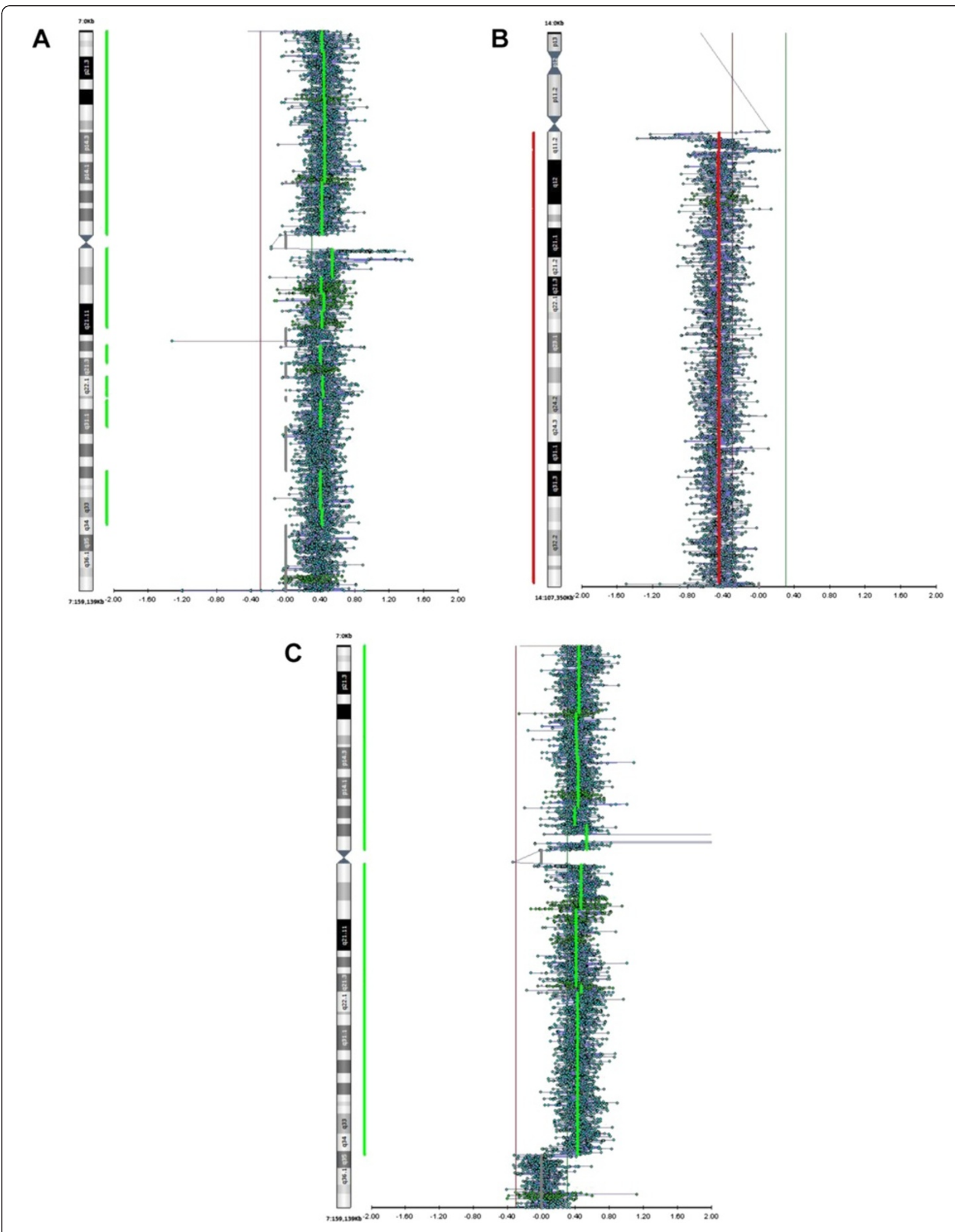

Figure 2 Validation of CNV Assay by array-CGH analysis. A) Trisomy of chromosome 7, but not EGFR amplification (probe position at +0.40 ), in BT314. B) Complete loss of chromosome 14q in BT314 (probe position at -0.40/-0.60); C) EGFR amplification in primary tumor BT418 (probe position at +0.60 ). 


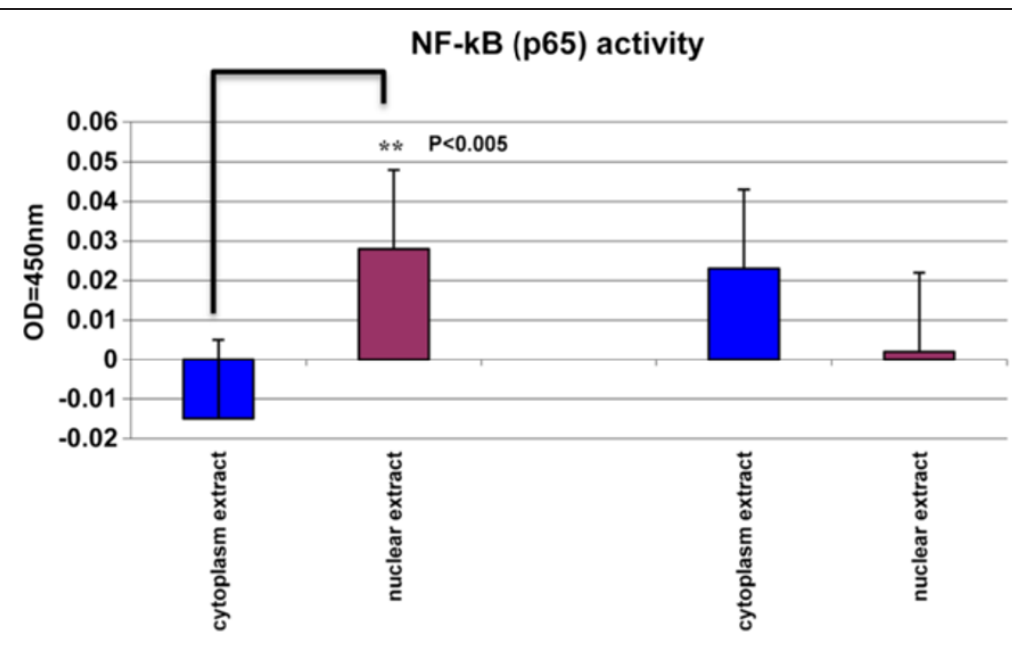

NFKBIA deleted NS
NFKBIA not deleted NS

Figure 3 Functional validation of NFKBIA heterozygous deletion in NS. Comparison of p65 activity in cytoplasmatic versus nuclear proteins in NS shows a significant (** $p<0.005)$ activity only in nuclear extract of NFKBIA deleted NS.

medium and NS in modified medium. In NS with complete medium all cell lines showed a marked decrease of EGFR amplification versus the corresponding BT, with 3 to 20 folds lower levels of EGFR/HGF copy number ratio. EGFR copy number was always higher in the absence of EGF: although this trend was present in all four cell lines, levels of EGFR amplification ranged from control values in NS 459 to levels higher than primary BT in NS 470 (Figure 5A).
Two cell lines, NS 462 and NS 470, showed heterozygous loss of NFKBIA in the complete medium, not detected in the original tumor: notably, the deletion was absent in the modified medium.

To further investigate the EGFR status we performed a quantitative evaluation of EGFR expression in $\mathrm{BT}$ and NS 462 and 470 by Real Time PCR, and investigated by a semi-quantitative method, RT-PCR, the presence of wild type EGFR and EGFRvIII (Figure 6A). The probe

\section{IL-6}

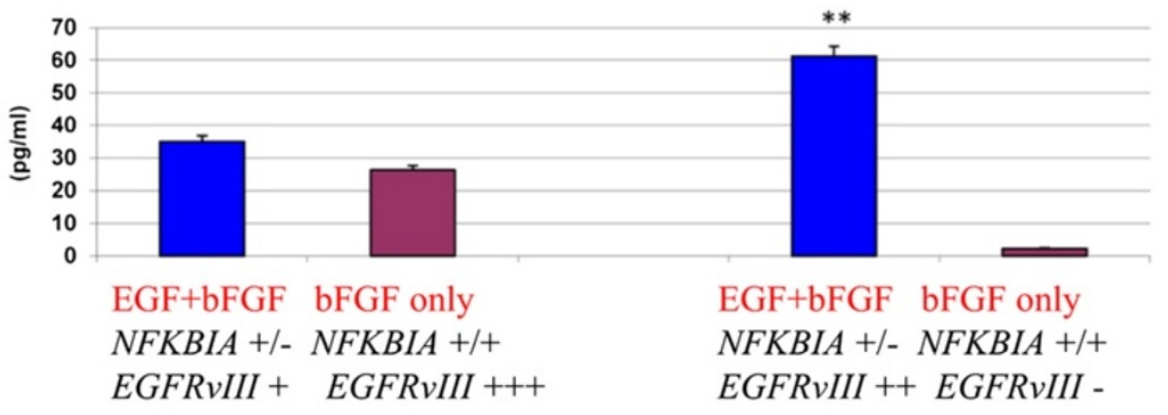

$462 \mathrm{NS}$

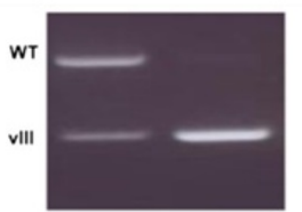

470 NS

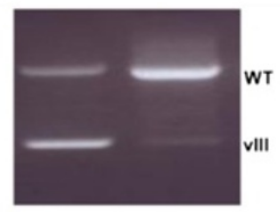

Figure 4 IL-6 expression in cultured cells. Quantification of IL-6 in surnatants of NS lines cultured in different conditions shows higher IL-6 production in BT470 in complete medium where NFKBIA deletion and EGFRvIII are both present $\left({ }^{* *} \mathrm{p}<0.001\right)$. RT-PCRs displaying wild type EGFR and EGFRvIll are shown below for comparison. 

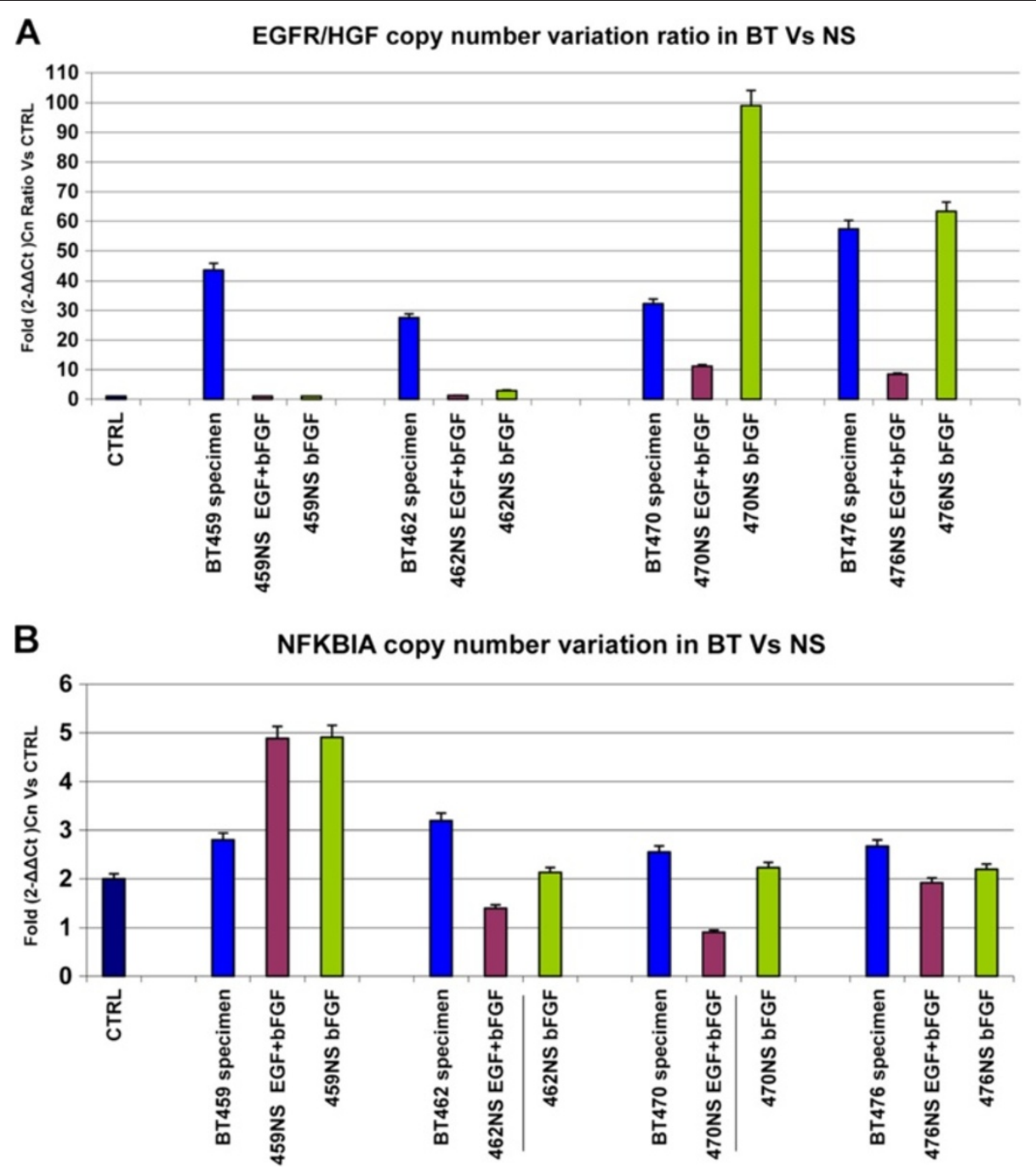

Figure 5 Analysis of copy number variations (CNVs) in BT and corresponding NS cultured in different conditions. A) EGFR status in NS cultured in presence of both growth factors and in presence of bFGF only shows a strong CNV decrease in complete medium and persistence of EGFR amplification at variable levels in absence of EGF. B) NFKBIA status in NS cultured in presence of both growth factors and in presence of bFGF alone shows an heterozygous NFKBIA deletion in two NS cultured in complete medium: BT462 and BT470.

used for Real-Time PCR recognized both wtEGFR and EGFRvIII (see also Methods). NS 462 showed increased presence of EGFRvIII and decreased presence of wtEGFR, particularly in the absence of EGF. BT470, on the contrary, showed a strong decrease of EGFRvIII in vitro and an increase of wtEGFR, particularly in the absence of EGF.

In Figure $6 \mathrm{~B}$ we show H\&E staining and immunohistochemistry with anti-EGFR antibody of BT462 and BT470 indicating strong EGFR expression, as expected.

Two other cell lines were also considered, BT463 and BT467 that did not show EGFR amplification or NFKBIA loss in the original tumor. Under both culture conditions (EGF plus bFGF or bFGF only) the status of these two genes did not change, suggesting that absence of EGF per se is not sufficient to drive EGFR amplification (Additional file 2: Figure S2).
Overall the data suggest that loss of EGFR amplification in vitro is favored by EGF presence in the culture medium. Clonal expansion of cells harboring NFKBIA deletion and/or EGFRvIII deletion may take place in this context.

\section{Survival analysis}

We looked for correlations of EGFR amplification and/ or NFKBIA deletion and overall survival (OS): four GBM were excluded from analysis as the patients died early after diagnosis for reasons unrelated to tumor progression. We could not find significant correlations between the presence of NFKBIA deletion in NS and OS (Additional file 3: Figure S3A) also considering the association with presence or lack of EGFR amplification (Additional file 3: Figure S3B). EGFR amplification was 

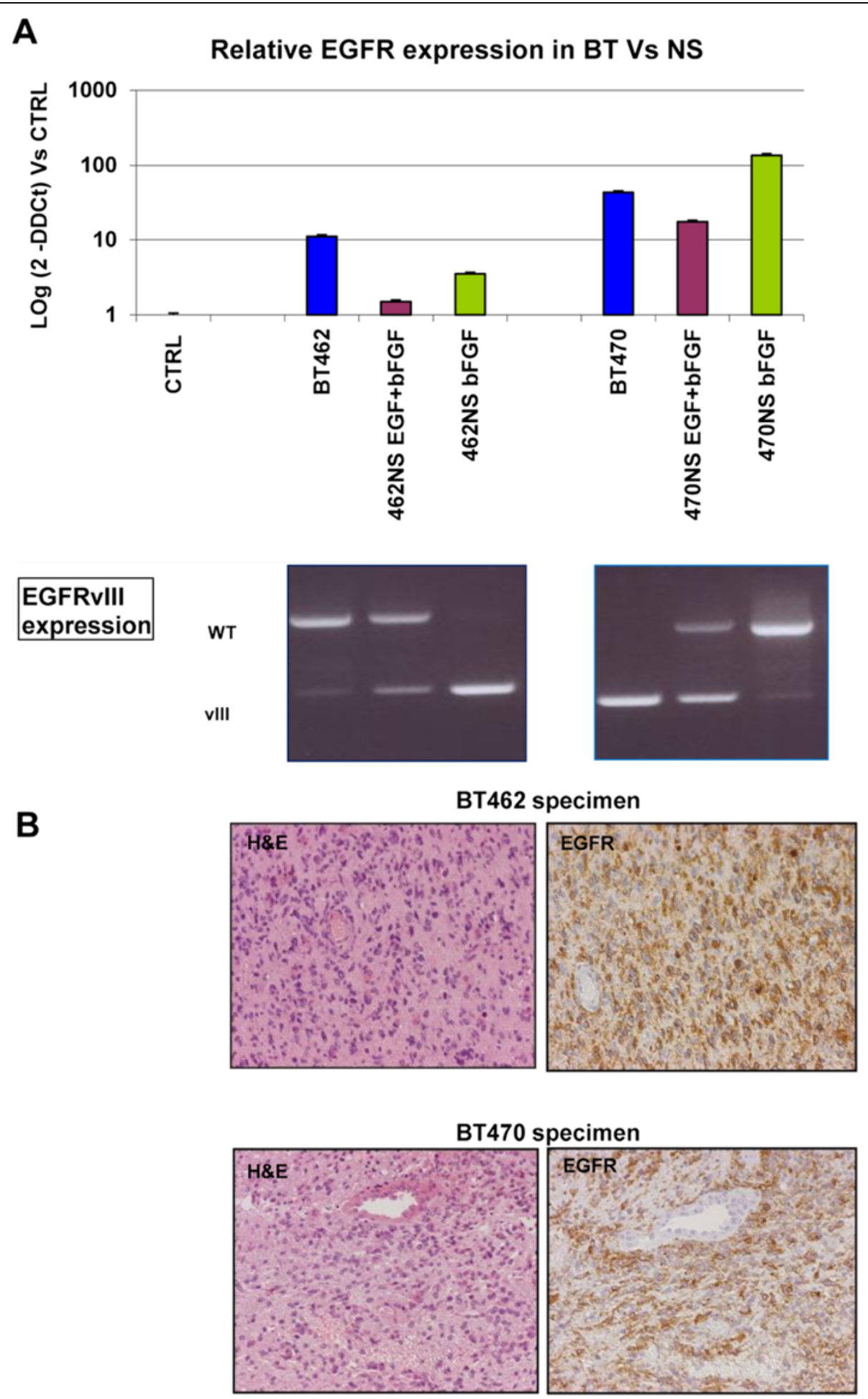

Figure 6 EGFR and EGFRvIll expression in primary BT and corresponding NS cultured in different conditions. A) The upper part shows mRNA levels of EGFR in primary tumors and corresponding NS; the lower part shows the presence of wild type EGFR and/or the mutant EGFRvIll: BT462 displays a gradual appearance of EGFRvIII in modified medium, whereas BT470 shows the presence of the mutant form in primary tumor and in NS cultured in complete medium; B) Immuno-histochemistry on tumors specimens shows high levels of EGFR in both tumors. 
also not associated with significant difference in OS (Additional file 3: Figure S3C), confirming ambiguities on the prognostic role of this marker [27].

\section{Discussion NFKBIA deletion is present at low frequency in primary GBM}

In this work we investigated the presence of the NFKBIA deletion in GBM. After the report by Bredel et al [19] one paper only reported on NFKBIA deletions in a fraction of GBM but did not confirm mutual exclusivity with EGFR amplification [28]. Recent reports based on TCGA data did not mention a high frequency of hemizygosity on 14q13, where NFKBIA maps $[29,30]$.

In our work we found a $4.3 \%$ frequency of NFKBIA deletion in 69 primary GBM. It is possible that the presence of normal cells in the surgical specimen leads to underestimation of the number of cases with deletion: this, however, seems plausible for a minority of cases. Furthermore, previous work reporting loss of heterozygosity on chromosome 14 showed loss of the entire $\mathrm{q}$ arm as a rather frequent event and partial losses located on 14q23-q32, telomeric to the NFKBIA locus [31,32].

It is plausible that other biological mechanisms independent of EGFR have a major role in NF-kB activation in GBMs. This family of transcription factors is the object of a number of studies pointing at its therapeutic targeting [33]. However its contribution to tumorigenesis in cells of neural origins needs further evaluation. Data from murine or rat NS showed that loss of both p65 and p50 NF-kB subunits results in a reduced number of progeny and in increased neuronal differentiation [34] and that activation of the canonical NF-kB pathway by TNF-alpha increases NS proliferation [35]. More recent data, however, suggest a pro-differentiation role of NF-kB. In murine embryonic stem cells Nanog, an essential factor for GBM tumorigenicity [36], binds to NF-kB proteins, inhibiting their transcriptional activity: overexpression of NF-kB proteins promoted differentiation, whereas inhibition increased expression of pluripotency markers [37]. Notably, also in GBM-NS the NF-kB transcriptional pathway was activated in the presence of serum-driven differentiation [38].

Overall, our first conclusion, based on this set of data is that the NFKBIA deletion is present but not frequent in primary GBM.

\section{NFKBIA deletion in culture is favored by EGF}

This observation is strengthened by the in vitro data we gathered on GBM neurospheres (NS). Unexpectedly, we found a large fraction of GBM NS showing results of the copy number assay for NFKBIA compatible with a heterozygous deletion. In parallel we observed the disappearance of EGFR amplification in part of these NS and decreased CNV values in others. Our results suggest the possibility that NFKBIA deletions are magnified (or created) in vitro in GBM NS that have decreased or lost amplification of EGFR. Since EGFR signaling may also impinge of NF-kB, as indicated by data in breast cancer [16] and GBMs [18], it seems plausible that in tumors "addicted" to this signaling pathway, NFKBIA deletions arise or are selected for in the presence of loss or decreased amplification of EGFR. The idea that part of the NFKBIA deletions in NS are favored by culture conditions and therefore do not mirror the actual situation of the primary tumor receives some indirect support by the lack of correlations with OS that we have found (Additional file 3: Figure S3), differently from what reported by Bredel et al [19].

Two sets of observations suggest that the assay we used is appropriate for CNV studies in these cells. First, we validated this technique by showing that HPRT1, a gene located in chromosome $\mathrm{X}$, is present with two copies in females and one copy in males (hemizygosity). Second, we showed that the decreased copy number of NFKBIA has functional consequences. We expected that decreased gene dosage of NFKBIA may cause increased migration to the nucleus of the NF-kB transcription factor and consequent increased activity of the p65 subunit. This was actually demonstrated in an ELISA-based testing on GBM NS with or without NFKBIA deletion. A third line of evidence was provided by array-CGH tested on one primary tumor, where NFKBIA deletion, detected by CNV assay, was associated to the entire loss of chromosome 14. EGFR amplification detected by CNV assay was also confirmed by array-CGH in these tumors and in one GBM NS. In this latter case, the NFKBIA deletion detected by the $\mathrm{CNV}$ assay was not found by array-CGH: we should consider, however, that the array $\mathrm{CGH}$ we used contain probes hybridizing to flanking and not intragenic regions of NFKBIA, so that hemizygosity would only be detected in the presence of large deletions or loss of the entire chromosome.

\section{EGFRvIll may be favored in vitro by lack of EGF}

The progressive lack of EGFR amplification in the presence of EGF in the culture medium is in good agreement with data from Schulte et al, showing that EGFR amplification is progressively lost under culture conditions used for NS growth [26]. Notably these authors found that EGFR amplification is associated to slower growth in vitro and that the difference in growth is decreased/canceled if EGF is removed from the culture medium [26]. The recent observation that EGFR amplification is associated to increased capacity for invasion rather than proliferation is in agreement with this observation [39].

Another response that can be favored in vitro in the absence of EGF-induced signaling by EGFR is the partial 
deletion of $E G F R$, as this implies the loss of the extracellular domain interacting with its ligand and consequent constitutive activation [12]. EGFRvIII may also activate NF-kB through mTORC2 [7]: thus, this deletion, that occurs in the majority of GBMs with EGFR amplification [40], may also preserve the function of the EGFR-NF-kB axis in glioma perpetuation.

Previous data by different groups including ours, have proposed that GBM NS provide a better system than serum-dependent cultures, to study GBM biology in vitro and in vivo [22,23,41]. Present results, however, indicate that presence of EGF may partially skew the molecular signatures present in primary GBMs. This is well illustrated by recent data showing that TNF-mediated activation of NF-kB is stimulated by mcroglia-macrophages in the tumor microenvironment and lost in vitro, thus favoring a proneural phenotype in GBM NS [42].

In conclusion, the data we obtained indicate that NFKBIA deletions are present but not frequent in GBMs, at difference with the initial report by Bredel et al [19]. This information and available evidence from the literature suggest that more work is required to thoroughly define the relevant mechanisms of NF-kB activation in GBM.

\section{Additional files}

Additional file 1: Figure S1. Copy number validation assay. Validation of copy number variation assay to assess hemizygous or heterozygous condition versus wild type genotype.

Additional file 2: Figure S2. Analysis of EGFR and NFKBIA copy number variations (CNVs) in primary tumors (BT) and corresponding neurospheres (NS) cultured in different conditions.

Additional file 3: Figure S3. Kaplan Meier analysis of overall survival (OS) of 65 patients with GBM with relationship to NFKBIA deletion and/ or EGFR amplification.

\section{Competing interests}

The authors declare that they have no competing interests.

\section{Authors' contributions}

MP performed CNV studies, investigated p65 activation and IL-6 expression and wrote preliminary results; PP, EB and SM cultured GB NS; GC performed the EGFR and EGFRvIII essays; VG, AR and FS performed CGH; ME provided clinical data; BP performed histological analysis; SP overviewed in vitro experiments; GF collabor ated to the study design and wrote the manuscript. All authors read and approved the manuscript.

\section{Acknowledgements}

We thank Dr Sergio Ottoleghi (University of Milan-Bicocca) and Dr Luigi Poliani (University of Brescia) for helpful discussion of the data.

\section{Author details}

'Molecular Neuro-Oncology Unit, IRCCS Foundation "C.Besta" Neurological Institute, via Celoria 11, 20133 Milan, Italy. ${ }^{2}$ Department of Experimental Oncology, IFOM-IEO Campus, via Adamello 16, 20139 Milan, Italy. ${ }^{3}$ Unit of Clinical Pathology and Medical Genetics, IRCCS Foundation "C.Besta" Neurological Institute, via Celoria 11, 20133 Milan, Italy. "Neuropathology Unit, IRCCS Foundation "C.Besta" Neurological Institute, via Celoria 11, 20133 Milan, Italy.
Received: 14 August 2013 Accepted: 28 November 2013

Published: 11 December 2013

\section{References}

1. Ohgaki H, Kleihues P: Epidemiology and etiology of gliomas. Acta Neuropathol 2005, 109:93-108.

2. Kotliarova S, Fine HA: Snapshot: glioblastoma multiforme. Cancer Cell 2012, 21:710-710. e1.

3. Phillips HS, Kharbanda S, Chen R, Forrest WF, Soriano RH, Wu TD, Misra A, Nigro JM, Colman H, Soroceanu L, Williams PM, Modrusan Z, Feuerstein BG, Aldape K: Molecular subclasses of high-grade glioma predict prognosis, delineate a pattern of disease progression, and resemble stages in neurogenesis. Cancer Cell 2006, 9:157-173.

4. Verhaak RGW, Hoadley KA, Purdom E, Wang V, Qi Y, Wilkerson MD, Miller CR, Ding L, Golub T, Mesirov JP, Alexe G, Lawrence M, O'Kelly M, Tamayo P, Weir BA, Gabriel S, Winckler W, Gupta S, Jakkula L, Feiler HS, Hodgson JG, James CD, Sarkaria JN, Brennan C, Kahn A, Spellman PT, Wilson RK, Speed TP, Gray JW, Meyerson M, et al: Integrated genomic analysis identifies clinically relevant subtypes of glioblastoma characterized by abnormalities in PDGFRA, IDH1, EGFR, and NF1. Cancer Cell 2010, 17:98-110.

5. Dunn GP, Rinne ML, Wykosky J, Genovese G, Quayle SN, Dunn IF, Agarwalla PK, Chheda MG, Campos B, Wang A, Brennan C, Ligon KL, Furnari F, Cavenee WK, Depinho RA, Chin L, Hahn WC: Emerging insights into the molecular and cellular basis of glioblastoma. Genes Dev 2012, 26:756-784.

6. Bonavia R, Inda MM, Vandenberg S, Cheng S-Y, Nagane M, Hadwiger P, Tan P, Sah DWY, Cavenee WK, Furnari FB: EGFRvIll promotes glioma angiogenesis and growth through the NF-KB, interleukin-8 pathway. Oncogene 2011, 31:4054-4066.

7. Tanaka K, Babic I, Nathanson D, Akhavan D, Guo D, Gini B, Dang J, Zhu S, Yang H, De Jesus J, Amzajerdi AN, Zhang Y, Dibble CC, Dan H, Rinkenbaugh A, Yong WH, Vinters HV, Gera JF, Cavenee WK, Cloughesy TF, Manning BD, Baldwin AS, Mischel PS: Oncogenic EGFR signaling activates an mTORC2NF-KB pathway that promotes chemotherapy resistance. Cancer Discov 2011, 1:524-538.

8. Jiang T, Grabiner B, Zhu Y, Jiang C, Li H, You Y, Lang J, Hung M-C, Lin X: CARMA3 is crucial for EGFR-Induced activation of NF-KB and tumor progression. Cancer Res 2011, 71:2183-2192.

9. Viatour P, Merville M-P, Bours V, Chariot A: Phosphorylation of NF-kappaB and IkappaB proteins: implications in cancer and inflammation. Trends Biochem Sci 2005, 30:43-52.

10. Bassères DS, Baldwin AS: Nuclear factor-kappaB and inhibitor of kappaB kinase pathways in oncogenic initiation and progression. Oncogene 2006, 25:6817-6830.

11. Mazzoleni S, Politi LS, Pala M, Cominelli M, Franzin A, Sergi Sergi L, Falini A, De Palma M, Bulfone A, Poliani PL, Galli R: Epidermal growth factor receptor expression identifies functionally and molecularly distinct tumor-initiating cells in human glioblastoma multiforme and is required for gliomagenesis. Cancer Res 2010, 70:7500-7513.

12. Huang HS, Nagane M, Klingbeil CK, Lin H, Nishikawa R, Ji XD, Huang CM, Gill GN, Wiley HS, Cavenee WK: The enhanced tumorigenic activity of a mutant epidermal growth factor receptor common in human cancers is mediated by threshold levels of constitutive tyrosine phosphorylation and unattenuated signaling. J Biol Chem 1997, 272:2927-2935.

13. Hegi ME, Rajakannu P, Weller M: Epidermal growth factor receptor: a re-emerging target in glioblastoma. Curr Opin Neurol 2012, 25:774-779.

14. Fan Q-W, Cheng CK, Gustafson WC, Charron E, Zipper P, Wong RA, Chen J, Lau J, Knobbe-Thomsen C, Weller M, Jura N, Reifenberger G, Shokat KM, Weiss WA: EGFR Phosphorylates Tumor-Derived EGFRvIII Driving STAT3/5 and Progression in Glioblastoma. Cancer Cell 2013, 24:438-449.

15. Lopez-Gines C, Gil-Benso R, Ferrer-Luna R, Benito R, Serna E, GonzalezDarder J, Quilis V, Monleon D, Celda B, Cerdá-Nicolas M: New pattern of EGFR amplification in glioblastoma and the relationship of gene copy number with gene expression profile. Mod Pathol 2010, 23:856-865.

16. Biswas DK, Cruz AP, Gansberger E, Pardee AB: Epidermal growth factor-induced nuclear factor kappa $B$ activation: a major pathway of cell-cycle progression in estrogen-receptor negative breast cancer cells. Proc Natl Acad Sci USA 2000, 97:8542-8547.

17. Yang W, Xia Y, Cao Y, Zheng Y, Bu W, Zhang L, You MJ, Koh MY, Cote G, Aldape K, Li Y, Verma IM, Chiao PJ, Lu Z: EGFR-induced and PKC $\varepsilon$ monoubiquitylation-dependent NF-KB activation upregulates PKM2 expression and promotes tumorigenesis. Mol Cell 2012, 48:771-784. 
18. Kapoor GS, Zhan Y, Johnson GR, O'Rourke DM: Distinct domains in the SHP-2 phosphatase differentially regulate epidermal growth factor receptor/NF-kappaB activation through Gab1 in glioblastoma cells. Mol Cell Biol 2004, 24:823-836.

19. Alvarez AA, SC B, Renfrow JJ, Chandler JP, Yu ILY, SC M, Carro MS, Ph D, Da F, Tagge MJ, Ferrarese R, Bredel C, Weyerbrock A, Vogel H, Dubner S, Mobley B, He X, Scheck AC, Iv GRH, Bredel M, Scholtens DM, Yadav AK, Phillips HS, Lukac PJ, Robe PA, Sikic BI, Aldape KD, Chakravarti A, Harsh GR: NFKBIA deletion in glioblastomas. N Engl J Med 2011, 364:627-637.

20. Yin D, Ogawa S, Kawamata N, Tunici P, Finocchiaro G, Eoli M, Ruckert C, Huynh T, Liu G, Kato M, Sanada M, Jauch A, Dugas M, Black KL, Koeffler HP: High-resolution genomic copy number profiling of glioblastoma multiforme by single nucleotide polymorphism DNA microarray. $\mathrm{Mol}$ Cancer Res 2009, 7:665-677.

21. Stiles CD, Rowitch DH: Glioma stem cells: a midterm exam. Neuron 2008, 58:832-846

22. Tunici P, Bissola L, Lualdi E, Pollo B, Cajola L, Broggi G, Sozzi G, Finocchiaro $\mathrm{G}$ : Genetic alterations and in vivo tumorigenicity of neurospheres derived from an adult glioblastoma. Mol Cancer 2004, 3:25.

23. Lee J, Kotliarova S, Kotliarov Y, Li A, Su Q, Donin NM, Pastorino S, Purow BW, Christopher N, Zhang W, Park JK, Fine HA: Tumor stem cells derived from glioblastomas cultured in bFGF and EGF more closely mirror the phenotype and genotype of primary tumors than do serum-cultured cell lines. Cancer Cell 2006, 9:391-403.

24. Pfaffl MW: A new mathematical model for relative quantification in realtime RT-PCR. Nucleic Acids Res 2001, 29:e45.

25. Inda M-M, Bonavia R, Mukasa A, Narita Y, Sah DWY, Vandenberg S, Brennan C, Johns TG, Bachoo R, Hadwiger P, Tan P, Depinho RA, Cavenee W, Furnar $\mathrm{F}$ : Tumor heterogeneity is an active process maintained by a mutant EGFR-induced cytokine circuit in glioblastoma. Genes Dev 2010, 24:1731-1745

26. Schulte A, Günther HS, Martens T, Zapf S, Riethdorf S, Wülfing C, Stoupiec M, Westphal M, Lamszus K: Glioblastoma stem-like cell lines with either maintenance or loss of high-level EGFR amplification, generated via modulation of ligand concentration. Clin Cancer Res 2012, 18:1901-1913.

27. Hobbs J, Nikiforova MN, Fardo DW, Bortoluzzi S, Cieply K, Hamilton RL, Horbinski C: Paradoxical relationship between the degree of EGFR amplification and outcome in glioblastomas. Am J Surg Pathol 2012, 36:1186-1193.

28. Idbaih A, Marie Y, Sanson M: NFKBIA deletion in glioblastomas. N Engl Med 2011, 365:277. author reply 277-8.

29. Frattini V, Trifonov V, Chan JM, Castano A, Lia M, Abate F, Keir ST, Ji AX, Zoppoli P, Niola F, Danussi C, Dolgalev I, Porrati P, Pellegatta S, Heguy A, Gupta G, Pisapia DJ, Canoll P, Bruce JN, McLendon RE, Yan H, Aldape K, Finocchiaro G, Mikkelsen T, Privé GG, Bigner DD, Lasorella A, Rabadan R, lavarone $A$ : The integrated landscape of driver genomic alterations in glioblastoma. Nat Genet 2013, 45:1141-1149.

30. Brennan CWW, Verhaak RGWGW, McKenna A, Campos B, Noushmehr H, Salama SRR, Zheng S, Chakravarty D, Sanborn JZZ, Berman SHH, Beroukhim R, Bernard B, Wu C-J, Genovese G, Shmulevich I, Barnholtz-Sloan J, Zou L, Vegesna R, Shukla SA, Ciriello G, Yung WKK, Zhang W, Sougnez C, Mikkelsen T, Aldape K, Bigner DDD, Van Meir EG, Prados M, Sloan A, Black KLL, et al: The somatic genomic landscape of glioblastoma. Cell 2013, 155:462-477.

31. Wooten EC, Fults D, Duggirala R, Williams K, Kyritsis AP, Bondy ML, Levin VA, O'Connell P: A study of loss of heterozygosity at 70 loci in anaplastic astrocytoma and glioblastoma multiforme with implications for tumor evolution. Neuro Oncol 1999, 1:169-176.

32. Hu J, Pang JC-S, Tong CY-K, Lau B, Yin X-L, Poon W-S, Jiang C-C, Zhou L-F, $\mathrm{Ng} \mathrm{H}-\mathrm{K}$ : High-resolution genome-wide allelotype analysis identifies loss of chromosome $14 \mathrm{q}$ as a recurrent genetic alteration in astrocytic tumours. Br J Cancer 2002, 87:218-224.

33. Nogueira L, Ruiz-Ontañon $P$, Vazquez-Barquero A, Moris F, Fernandez-Luna $J$ : The NFKB pathway: a therapeutic target in glioblastoma. Oncotarget 2011, 2:646-653.

34. Young KM, Bartlett PF, Coulson EJ: Neural progenitor number is regulated by nuclear factor-kappaB p65 and p50 subunit-dependent proliferation rather than cell survival. J Neurosci Res 2006, 83:39-49.

35. Widera D, Mikenberg I, Elvers M, Kaltschmidt C, Kaltschmidt B: Tumor necrosis factor alpha triggers proliferation of adult neural stem cells via IKK/NF-kappaB signaling. BMC Neurosci 2006, 7:64
36. Zbinden M, Duquet A, Lorente-Trigos A, Ngwabyt S-N, Borges I: Ruiz i Altaba A: NANOG regulates glioma stem cells and is essential in vivo acting in a cross-functional network with GLI1 and p53. EMBO J 2010, 29:2659-2674

37. Torres J, Watt FM: Nanog maintains pluripotency of mouse embryonic stem cells by inhibiting NFkappaB and cooperating with Stat3. Nat Cell Biol 2008, 10:194-201.

38. Nogueira L, Ruiz-Ontañon P, Vazquez-Barquero A, Lafarga M, Berciano MT, Aldaz B, Grande L, Casafont I, Segura V, Robles EF, Suarez D, Garcia LF, Martinez-Climent JA, Fernandez-Luna JL: Blockade of the NFkB pathway drives differentiating glioblastoma-initiating cells into senescence both in vitro and in vivo. Oncogene 2011, 30:3537-3548.

39. Talasila KM, Soentgerath A, Euskirchen P, Rosland GV, Wang J, Huszthy PC, Prestegarden L, Skaftnesmo KO, Sakariassen PØ, Eskilsson E, Stieber D, Keunen O, Brekka N, Moen I, Nigro JM, Vintermyr OK, Lund-Johansen M, Niclou S, Mørk SJ, Enger PO, Bjerkvig R, Miletic H: EGFR wild-type amplification and activation promote invasion and development of glioblastoma independent of angiogenesis. Acta Neuropatho/ 2013, 125:683-698.

40. Furnari FB, Fenton T, Bachoo RM, Mukasa A, Stommel JM, Stegh A, Hahn WC, Ligon KL, Louis DN, Brennan C, Chin L, DePinho RA, Cavenee WK: Malignant astrocytic glioma: genetics, biology, and paths to treatment. Genes Dev 2007, 21:2683-2710.

41. De Bacco F, Casanova E, Medico E, Pellegatta S, Orzan F, Albano R, Luraghi $P$, Reato $G$, D'Ambrosio A, Porrati P, Patanè M, Maderna E, Pollo B, Comoglio PM, Finocchiaro G, Boccaccio C, Patane M: The MET oncogene is a functional marker of a glioblastoma stem cell subtype. Cancer Res 2012, 72:22738909.

42. Bhat KPL, Balasubramaniyan V, Vaillant B, Ezhilarasan R, Hummelink K, Hollingsworth F, Wani K, Heathcock L, James JD, Goodman LD, Conroy S, Long L, Lelic N, Wang S, Gumin J, Raj D, Kodama Y, Raghunathan A, Olar A, Joshi K, Pelloski CE, Heimberger A, Kim SH, Cahill DP, Rao G, Den Dunnen WFA, Boddeke HWGM, Phillips HS, Nakano I, Lang FF, et al: Mesenchymal differentiation mediated by NF-KB promotes radiation resistance in glioblastoma. Cancer Cell 2013, 24:331-346.

doi:10.1186/1476-4598-12-160

Cite this article as: Patanè et al:: Frequency of NFKBIA deletions is low in glioblastomas and skewed in glioblastoma neurospheres. Molecular Cancer 2013 12:160.

\section{Submit your next manuscript to BioMed Central and take full advantage of:}

- Convenient online submission

- Thorough peer review

- No space constraints or color figure charges

- Immediate publication on acceptance

- Inclusion in PubMed, CAS, Scopus and Google Scholar

- Research which is freely available for redistribution 